\title{
Screening for diabetes
}

\section{Targeted screening causes less stress than mass screening, but there is insufficient evidence to advocate either}

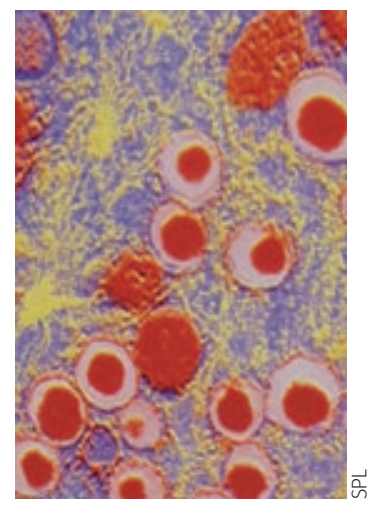

RESEARCH, pp 486, 490

\section{Ronald P Stolk professor of} clinical epidemiology, Department of Epidemiology, University Medical Centre Groningen, University of Groningen, PO Box 30.001, 9700 RB Groningen, Netherlands r.p.stolk@epi.umcg.nl Competing interests: None declared.

Provenance and peer review: Commissioned; not externally peer reviewed.

BMJ 2007;335:457-8 doi: 10.1136/bmj.39323.395336.BE
In this week's BMJ, two studies by Eborall and colleagues assess the psychological impact of screening for diabetes mellitus in primary care. ${ }^{12}$ Screening for diabetes is primarily aimed at preventing cardiovascular disease. The risk of cardiovascular disease increases proportionally as concentrations of glucose increase, with no threshold below which the risk remains constant. ${ }^{3}$ This is in contrast with other complications of diabetes-such as retinopathy, neuropathy, and nephropathy-the risk of which sharply increases when glucose concentrations exceed the threshold of $11 \mathrm{mmol} / \mathrm{l}$. At this concentration the typical diagnostic signs of diabetes are usually present-thirst, polyuria, and weight loss.

Population based mass screening for diabetes has been proposed for several years. ${ }^{4}$ This has been fuelled by rising plasma glucose concentrations in most populations worldwide, as a result of increasing body weight associated with a more sedentary lifestyle and changes in diet. Arguments for mass screening include the fact that mildly increased plasma glucose does not cause symptoms and usually persists for several years, it can be determined by a capillary drop of blood, and it is associated with increased risk of cardiovascular disease. ${ }^{5}$

However, two questions need to be resolved before screening can be recommended. Firstly, will treating asymptomatic hyperglycaemia help prevent cardiovascular disease? Secondly, what psychological harm could be caused by anxiety regarding the screening result or the effects of a diagnosis that requires changes in diet and activity patterns and lifelong use of drugs? ${ }^{6}$ The first question should be answered by the ongoing international ADDITION trial into the effectiveness of multifactorial treatment for cardiovascular disease in people with screen detected diabetes. ${ }^{7}$

The two studies in this week's issue look at the second question, at least in white British people. Eborall and colleagues studied the psychological effects of stepwise screening programme for diabetes conducted within the Cambridge arm of the ADDITION trial. They found no changes in anxiety, depression, or worries about diabetes between the invitation for the first screening visit and a year after completing the programme. ${ }^{1}$ Moreover, the results did not differ significantly between people invited for screening and those who were not invited.

The authors also conducted a smaller qualitative study within the Cambridge ADDITION study at several time points during the stepwise screening process. ${ }^{2}$ Participants seemed to adapt their feelings about the possibility of having a chronic disease with each step in the screening process, thereby controlling the psychological burden. ${ }^{2}$ Both studies were hampered by a low response rate, which is probably not random. If people who were anxious about examinations, forms, and medical or research staff preferentially chose not to participate, a psychological harm of screening would be masked by selection bias. However, this is unlikely because the results are in line with the absence of psychological effects of blood pressure screening ${ }^{8}$ and with a smaller study in the Dutch part of the ADDITION trial. ${ }^{9}$

It is important to realise that the ADDITION study consisted of a stepwise screening procedure. Only people with an increased risk of diabetes-on the basis of a raised cardiovascular risk known to their general practitioner-were invited for the first examination. They had probably been counselled about their weight or were aware of their increased risk of cardiovascular disease because they had been prescribed drugs to lower their blood pressure. As Eborall and colleagues suggest, ${ }^{2}$ this might explain the limited psychological stress of the screening procedure and a diagnosis of diabetes-people were already aware of their high risk before the invitation for screening. Harmful psychological effects are more likely to occur in population based mass screening, where everybody within a certain age range is invited for a plasma glucose measurement (like breast cancer screening). In that situation, the stepwise approach is replaced by one large leap from feeling completely healthy to harsh lifestyle changes and lifelong use of drugs.

Targeted screening for diabetes, also referred to as case finding or opportunistic screening, means that health professionals measure glucose in people with a presumed increased risk of diabetes. Eborall and colleagues' results further tip the balance in favour of individual targeted screening over mass population screening for diabetes. Screening and diagnosis within the healthcare setting enables individually tailored follow-up and treatment.

As Eborall and colleagues have shown such a stepwise approach may be important to minimise psychological stress. ${ }^{2}$ Targeted screening is also more cost effective than population screening. ${ }^{10}$

Nevertheless, even though targeted screening may not increase psychological stress we still need to show that treating asymptomatic people with slightly raised glucose concentrations is effective. At the moment there is insufficient evidence to recommend screening for 
diabetes, and until the results of the ADDITION trial are known we will have to wait until people present with the classic symptoms of thirst and polyuria before screening them.

1 Eborall HC, Griffin SJ, Prevost AT, Kinmonth A-L, French DP, Sutton S. Psychological impact of screening for type 2 diabetes: controlled trial and comparative study embedded in the ADDITION Cambridge randomised controlled trial. BMJ 2007 doi: 10.1136/ bmj.39303.723449.55.

2 Eborall H, Davies R, Kinmonth A-L, Griffin S, Lawton J. Patients' experiences of screening for type 2 diabetes: prospective qualitative study embedded in the ADDITION Cambridge randomised trial. BMJ 2007 doi: 10.1136/bmj.39308.392176.BE.

3 Coutinho M, Gerstein HC, Wang Y, YusufS. The relationship between glucose and incident cardiovascular events. A metaregression analysis of published data from 20 studies of 95,783 individuals followed for 12.4 years. Diabetes Care 1999;22:233-40.

4 Engelgau MM, Narayan KM, Herman WH. Screening for type 2 diabetes. Diabetes Care 2000;23:1563-80.

5 Jansen PGM, Gorter KI, Stolk RP, Rutten GEHM. Screen detected subjects with type 2 diabetes and impaired glucose tolerance have more adverse cardiovascular risk than subjects with impaired fasting glucose especially when they are obese. The ADDITION Netherlands study. Prim Care Diabet 2007;1:69-74.

6 Wareham NJ, Griffin SJ. Should we screen for type 2 diabetes? Evaluation against National Screening Committee criteria. BMJ 2001;322:986-8.

7 Lauritzen T, Griffin S, Borch-Johnsen K, Wareham NJ, Wolffenbuttel BH, Rutten G. The ADDITION study: proposed trial of the cost-effectiveness of an intensive multifactorial intervention on morbidity and mortality among people with type 2 diabetes detected by screening. Int J Obes Relat Metab Disord 2000;24(suppl 3):S6-11.

8 Sheridan S, Pignone M, Donahue K. Screening for high blood pressure: a review of the evidence for the US preventive services task force. $\mathrm{Am}$ ] PrevMed 2003;25:151-8.

9 Thoolen BJ, de Ridder DT, Bensing JM, Gorter KJ, Rutten GE. Psychological outcomes of patients with screen-detected type 2 diabetes: the influence of time since diagnosis and treatment intensity. Diabetes Care 2006;29:2257-62.

10 Glumer C, Yuyun M, Griffin S, Farewell D, Spiegelhalter D, Kinmonth AL, et al. What determines the cost-effectiveness of diabetes screening? Diabetologia 2006;49:1536-44.

\section{RESEARCH, pp 493, 497}

\section{Frank J Snoek professor o}

medical psychology, Diabetes

Psychology Research Group, VU

University Medical Centre, 1081

BT Amsterdam, Netherlands

fj.snoek@vumc.nl

Competing interests: None

declared.

Provenance and peer review:

Commissioned; not externally peer

reviewed.

BMJ 2007;335:358-9

doi: 10.1136/bmj.39315.443160.BE

\section{Self management of type 2 diabetes}

\section{More efforts are needed to capture the patients' perspective}

The introduction of home blood glucose monitoring in the late 1970s was instrumental in shifting the focus of the management of diabetes from doctors to patients. ${ }^{1}$ It is now a common view that patients are primarily responsible for the daily management of their diabetes, which includes self monitoring, at least in patients treated with insulin. The usefulness of self monitoring in patients with type 2 diabetes not treated with insulin is controversial, a debate that was recently fuelled by findings from the DIGEM trial. ${ }^{2}$

Two studies in this week's $B M J$ are related to optimising the treatment of type 2 diabetes. ${ }^{34}$ In the first, Peel and colleagues ${ }^{3}$ report the results from a longitudinal qualitative study of the views of patients with type 2 diabetes about self monitoring, using a repeat interview design. The authors rightly point out that the patient's view has been largely absent in discussions on self monitoring in type 2 diabetes. While self testing of blood glucose has the potential to empower patients, it is often viewed as complex and inconvenient. Finger pricks can be painful, and the repeated confrontation with unexpected outcomes and "bad" results can lead to frustration, guilt, and indeed "learned helplessness." 56 These negative effects on patients' wellbeing are probably responsible to a large extent for the low adherence to self monitoring seen in patients with both type 1 and type 2 diabetes. $^{7}$

Peel and colleagues previously reported from the same research project on patients views on self monitoring six months after diagnosis. ${ }^{8}$ Results suggested that patients with poorly controlled diabetes were more likely than those with good control to voice concerns and to have problems with self monitoring.

Three years after diagnosis, 18 of these patients (one on insulin) who had ever self monitored their blood glucose were contacted for a third interview round, to explore (changes in) their experiences and views of self monitoring. ${ }^{3}$ The relevance of a longitudinal approach is underscored by recent research showing that self monitoring practices change over time and may have different effects on glycaemic control in new and established users. ${ }^{9}$ Peel and colleagues found that fewer patients were self monitoring over time, and those who did continue monitoring did so less frequently. Some patients expressed uncertainty about the meaning of the test results and how to act on them, while others found self monitoring to be reassuring and did it routinely. Most participants voiced concerns about the value health professionals placed on their readings. Doctors generally appeared to show little interest in patients' test results after the initial phase, leading some patients to perceive self monitoring as not very important or even pointless. Interestingly, some patients continued to self monitor despite lack of guidance and the perceived health professionals' disinterest.

The generalisability of the findings from this small qualitative study remains uncertain, although the reported experiences and attitudes seem all too realistic and common. The study reminds us of the importance of demographic, social, and psychological variables in explaining the observed interindividual differences. The question of how useful self monitoring is in patients not being treated with insulin remains, but clearly Peel and colleagues' study confirms the need to develop educational strategies that can help patients effectively use blood glucose monitoring and manage negative feedback. As the authors point out, self monitoring is apparently still surrounded with feelings of personal failure and self blame, particularly in female patients. Experience has shown that simply providing patients with a manual on how to overcome common emotional and behavioural barriers to self testing can have significant beneficial effects on psychology and glycaemic control. ${ }^{10}$ The second study, a systematic review by Eurich and colleagues, assesses the optimum drug treatment for patients with type 2 diabetes and 
heart failure. ${ }^{4}$ The review used evidence from eight studies to look at the effects of various blood glucose lowering drugs, including oral drugs and insulin, on morbidity and mortality in patients with type 2 diabetes and heart failure. It found that metformin is the only antidiabetic drug that is not associated with any measurable harm in people with diabetes and heart failure. In fact, metformin was associated with reduced mortality.

A weakness of the review is that most of the data were observational and only one randomised trial was included. However, the review does complement a recently published systematic review on the effectiveness and safety of oral drugs for type 2 diabetes, which found that metformin and second generation sulphonylureas are similarly effective or even superior in terms of glycaemic effects to newer and more expensive agents. ${ }^{11}$

Despite the new information provided by these reviews, more evidence is urgently needed on the benefits of newer antidiabetic drugs in different populations of patients. Future trials should not only include clinical end points, but also outcomes that are important to patients, in line with the draft guidance from the US Food and Drug Administration. ${ }^{12}$ Patients are experts on their own quality of life. It would seem wise therefore for the medical industry and scientists to ask patients to join them in designing clinical trials and choosing the most relevant outcomes to be reported by patients.
1 Saudek CD, Derr RL, Kalyani RR. Assessing glycemia in diabetes using self-monitoring blood glucose and hemoglobin A1c. JAMA 2006;293:1688-97.

2 Farmer A, Wade A, Goyder E, Yudkin P, French D, Craven A, et al. Impact of self monitoring of blood glucose in the management of patients with non-insulin treated diabetes: open parallel group randomized trial. BMJ 2007;335:132-6.

3 Peel E, Douglas M, Lawton J. Self monitoring of blood glucose in type 2 diabetes: longitudinal qualitative study of patients' perspectives. BM/ 2007 doi: 10.1136/bmj.39302.444572.DE.

4 Eurich DT, McAlister FA, Blackburn DF, Majumdar SR, Tsuyuki RT, Varney J, et al. Benefits and harms of antidiabetic agents in patients with diabetes and heart failure: systematic review. BMJ 2007 doi: 10.1136/bmj.39314.620174.80.

5. Dunn SM. Psychological issues in diabetes management: blood glucose monitoring and learned helplessness. Pract Diabetes 1987;4:108-10.

6 Wysocki T. Impact of blood glucose monitoring on diabetic control: obstacles and interventions. J Behav Med 1999;12:183-203.

7 Vincze G, Barner JC, Lopze D. Factors associated with adherence to self-monitoring of blood glucose among persons with diabetes. Diabetes Educ 2004;30:112-25.

8 Peel E, Parry O, Douglas M, Lawton J. Blood glucose self-monitoring in non-insulin treated type 2 diabetes: a qualitative study of patients' perspectives. BrJ Gen Pract 2004;54:183-8.

9 Karter AJ, Parker MM, Moffet HH, Spence MM, Chan J, Ettner SL, et al. Longitudinal study of new and prevalent use of self-monitoring of blood glucose. Diabetes Care 2006;29:1757-63.

10 Moreland EC, Volkering LK, Lawlor MT, Chalmers KA, Anderson BJ, Laffel LM. Use of a blood glucose monitoring manual to enhance monitoring adherence in adults with diabetes. A randomized controlled trial. Arch Intern Med 2006;166:689-95.

11 Bolen S, Feldman L, Vassy J, Wilson J, Yeh HC, Marinopoulos S, et al. Systematic review: comparative effectiveness and safety of oral medications for type 2 diabetes mellitus. Ann Intern Med 2007; Jul 16; Epub ahead of print.

12 Food and Drug Administration. Guidance for industry: patient reported outcome measures. Use in medical product development to support label claims. Draft guidance. February 2006. www.fda.gov/ cder/guidance/5460dft.pdf.

\section{Mild hypothermia for post cardiac arrest syndrome Is recommended by evidence based guidelines yet uptake remains poor}

\section{Jasmeet Soar consultant in \\ anaesthesia and intensive care medicine, Southmead Hospital, North Bristol NHS Trust, Bristol BS10 5NB \\ Jasmeet.soar@nbt.nhs.uk Jerry P Nolan consultant in anaesthesia and intensive care medicine, Royal United Hospital, Bath BA1 3NG \\ Competing interests: JS is vice chairman of the Resuscitation Council UK. JPN is chairman of the Resuscitation Council UK and co- chair of the International Liaison Committee on Resuscitation. JS and IPN are involved in writing international and national guidelines for cardiopulmonary resuscitation.}

Provenance and peer review: Commissioned; not externally peer reviewed.

BMJ 2007;335:459-60 doi:10.1136/bmj.39315.519201.BE
Most people who have a cardiac arrest die. Many who are resuscitated subsequently die over the next few hours or days, and those who survive are at risk of cognitive dysfunction. This gloomy reality has prompted research into interventions to improve the prognosis of cardiac arrest; one of these interventions is the induction of mild hypothermia after spontaneous circulation has been restored.

Apart from patients resuscitated from a very brief cardiac arrest, most survivors will be comatose initially, and those without extensive comorbidity will be admitted to an intensive care unit. Unconscious, mechanically ventilated survivors of cardiac arrest account for one in 17 of all admissions to intensive care in the United Kingdom. ${ }^{1}$ A third of these patients survive to hospital discharge. In one centre, two thirds of deaths in intensive care in initial survivors of cardiac arrest that occurred out of hospital, and a quarter of deaths after cardiac arrest that occurred in hospital, were attributed primarily to a neurological cause. ${ }^{2}$

Organ injury caused by ischaemia and hypoxia during prolonged cardiac arrest is compounded by reperfusion injury that occurs when a spontaneous circulation is restored. These insults trigger a systemic inflammatory response, similar to that associated with sepsis. ${ }^{3}$ The term post cardiac arrest syndrome describes this systemic response and associated multiple organ dysfunction.

Mild hypothermia is neuroprotective both before and after brain ischaemia through several mechanisms, including reduced production of excitotoxins and free radicals, suppression of apoptosis, and other antiinflammatory actions. ${ }^{4}$ Animal studies show that hypothermia is more effective the earlier it is started after return of spontaneous circulation. Two randomised but unblinded clinical trials ${ }^{56}$ and a meta-analysis ${ }^{7}$ show improved survival and neurological outcome in adults who remained comatose after initial resuscitation from out of hospital ventricular fibrillation cardiac arrest, and who were cooled within minutes to hours after initial resuscitation. Patients were cooled to $33^{\circ} \mathrm{C}^{6}$ or to $32-34^{\circ} \mathrm{C}$ for $12-24$ hours. ${ }^{5}$ Between four and 13 patients need to be treated for one extra survivor to leave hospital with good neurological function. ${ }^{7}$ None of the studies showed an increase in the number of survivors with poor neurological function.

Contraindications to inducing mild hypothermia include severe systemic infection, pre-existing coagulopathy (previous thrombolytic therapy is not a contraindication), and established multiple organ 
failure. Complications of mild hypothermia include increased infection, cardiovascular instability, coagulopathy, hyperglycaemia, increased plasma amylase, hypophosphataemia, and hypomagnesaemia. ${ }^{8}$ Most of these complications are easy to treat in the intensive care unit or can be reduced by raising the patient's temperature slowly by $1-2^{\circ} \mathrm{C}$.

Despite the evidence and inclusion in guidelines, the uptake of mild hypothermia by intensive care units around the world is poor. ${ }^{9}$ Some clinicians remain sceptical about the evidence-just two relatively small unblinded controlled trials; one used pseudorandomisation to allocate treatments, ${ }^{6}$ the other enrolled just $8 \%$ of all the patients assessed for eligibility. ${ }^{5}$

It is still not clear whether patients outside of the inclusion criteria used in the original trials would benefit from hypothermia (such as those with cardiac arrests occurring in hospital or non-ventricular fibrillation cardiac arrests). A randomised trial of hypothermia after resuscitation following in hospital cardiac arrest is ongoing (ClinicalTrials.gov identifier NCT00457431).

An effective and easy method to initiate cooling is rapid infusion of cold $\left(4^{\circ} \mathrm{C}\right)$ intravenous fluid. ${ }^{10}$ Cooling should start as soon as possible. This means that for cardiac arrests occurring out of hospital, cooling should ideally be started at the scene of the arrest or in the ambulance or, at the very latest, in the emergency department. A randomised controlled trial assessing long term survival after prehospital induction of hypothermia with cold intravenous fluid is about to start (ClinicalTrials.gov identifier NCT00391469).

No consensus exists on the best way to maintain hypothermia, the optimum duration for hypothermia, or how best to rewarm the patient. In our experience, simple methods to maintain hypothermia-such as surface cooling with icepacks and fans-can work, but over-cooling and under-cooling are common. Intravascular cooling methods enable tighter temperature control, but insertion of large bore intravascular catheters has risks and the disposables are expensive.

Mild hypothermia is just one component of treatment for the post cardiac arrest syndrome. Other important components include early coronary reperfusion (percutaneous intervention or thrombolysis), controlled ventilation to achieve normal arterial blood oxygen and carbon dioxide tensions, cardiovascular support with vasoactive drugs, and, if necessary, an intra-aortic balloon pump, and intensive control of blood glucose. Evidence suggests that implementing a systematic treatment protocol after resuscitation improves outcomes. ${ }^{11}$

Current guidelines recommend that unconscious adults with spontaneous circulation after out of hospital cardiac arrest should be cooled to $32-34^{\circ} \mathrm{C}$ for $12-24$ hours when the initial rhythm was ventricular fibrillation. ${ }^{12}$ This treatment may be considered for unconscious adult patients with spontaneous circulation after out of hospital cardiac arrest with any other rhythm or after in hospital cardiac arrest. Many intensive care doctors, including ourselves, now cool most comatose patients admitted to intensive care after cardiac arrest.

1 Nolan JP, Laver SR, Welch CA, Harrison DA, Gupta V, Rowan K. Outcome following admission to UK intensive care units after cardiac arrest: a secondary analysis of the ICNARC case mix programme database. Anaesthesia 2007 (in press)

2 Laver S, Farrow C, Turner D, Nolan J. Mode of death after admission to an intensive care unit following cardiac arrest. Intensive Care Med 2004:30:2126-8.

3 Adrie C, Adib-Conquy M, Laurent I, Monchi M, Vinsonneau C, Fitting C, et al. Successful cardiopulmonary resuscitation after cardiac arrest as a "sepsis-like" syndrome. Circulation 2002;106:562-8.

4 Gunn AJ, Thoresen M. Hypothermic neuroprotection. NeuroRx 2006;3:154-69.

5 Hypothermia After Cardiac Arrest Study Group. Mild therapeutic hypothermia to improve the neurologic outcome after cardiac arrest. $N$ Engl J Med 2002;346:549-56.

6 Bernard SA, Gray TW, Buist MD, Jones BM, Silvester W, Gutteridge G, et al. Treatment of comatose survivors of out-of-hospital cardiac arrest with induced hypothermia. N Engl J Med 2002;346:557-63.

7 Holzer M, Bernard SA, Hachimi-Idrissi S, Roine RO, Sterz F, Mullner M. Hypothermia for neuroprotection after cardiac arrest: systematic review and individual patient data meta-analysis. Crit Care Med 2005;33:414-8.

8 Polderman KH. Application of therapeutic hypothermia in the intensive care unit. Opportunities and pitfalls of a promising treatment modality. Part 2: Practical aspects and side effects. Intensive Care Med 2004;30:757-69.

9 Merchant RM, Soar J, Skrifvars MB, Silfvast T, Edelson DP, Ahmad F, et al. Therapeutic hypothermia utilization among physicians after resuscitation from cardiac arrest. Crit Care Med 2006;34:1935-40.

10 Kim F, Olsufka M, Longstreth WT Jr, Maynard C, Carlbom D, Deem S, et al. Pilot randomized clinical trial of prehospital induction of mild hypothermia in out-of-hospital cardiac arrest patients with a rapid infusion of 4 degrees C normal saline. Circulation 2007;115:3064-70.

11 Sunde K, Pytte M, Jacobsen D, Mangschau A, Jensen LP, Smedsrud C, et al. Implementation of a standardised treatment protocol for post resuscitation care after out-of-hospital cardiac arrest. Resuscitation 2007;73:29-39.

12 Nolan IP, Morley PT, Vanden Hoek TL, Hickey RW. Therapeutic hypothermia after cardiac arrest. An advisory statement by the advanced life support task force of the International Liaison Committee on Resuscitation. Resuscitation 2003;57:231-5.

\section{HIV phylogenetics}

\section{Criminal convictions relying solely on this to establish transmission are unsafe}

\section{Deenan Pillay professor of} virology, University College London, London W1T 4JF and Centre for Infections, Health

Protection Agency, London NW9 5EQ

d.pillay@ucl.ac.uk

(see next page for co-authors)
The recent flurry of criminal cases brought against people in the United Kingdom accused of infecting their sexual partner(s) with HIV has resulted in several convictions. This has caused concern among health professionals and community groups about the detrimental effect such cases may have on disclosure of HIV infection and uptake of voluntary HIV testing, which contrasts with the move to normalise HIV test- ing and clinical care. The potential negative effect of this on the public health programme to reduce transmission of HIV has been widely discussed in these pages $^{12}$ and elsewhere. ${ }^{3}$

Virological evidence, specifically HIV gene sequence data obtained from the defendant and complainant, has been used in these cases because a prerequisite for establishing criminal liability is that the 
Andrew Rambaut senior research fellow, Institute of Evolutionary Biology, University of Edinburgh, Edinburgh EH93JT Anna Maria Geretti consultant medical virologist, Royal Free Hospital, London NW3 2PF Andrew J Leigh Brown professor of evolutionary genetics, Institute of Evolutionary Biology, University of Edinburgh, Edinburgh EH9 3JT

Competing interests: This article takes account of a discussion

held by the UK Expert Advisory Group on AIDS (EAGA; www advisorybodies.doh.gov.uk/

eaga/). DP is a member of EAGA $A L B, A M G$, and DP have acted as expert witnesses in relation to criminal transmission cases in recent years.

Provenance and peer review: Not commissioned; externally peer reviewed.

BMJ 2007;335:460-61 doi: 10.1136/bmj.39315.398843.BE defendant caused the complainant's infection. Because HIV-1, like other RNA viruses, evolves rapidly, the virus isolated from independently infected people is typically distinct. The extent of similarity between viruses from different people is associated with the likelihood of a common source of infection.

Within infected people, viral populations expand from a small initial population, and the viral genome can change during chronic infection by up to $1 \%$ each year. Thus, an onward transmission, if it occurred some time after initial infection, is likely to involve a strain distinguishable from the host viral strain. Viral phylogenetics, the scientific study of evolutionary relations between strains, has previously been used successfully to explore occupational and healthcare related transmissions in the context of detailed epidemiological information in cases in Florida, ${ }^{4}$ Baltimore ${ }^{5}$ and Louisiana. ${ }^{6}$

In recent criminal cases in the UK, attempts have been made to present evidence on the viral sequence as a conceptual framework akin to DNA fingerprinting (for which the odds of DNA samples from two different people being the same is typically lower than the population of the planet). In our view, this analogy is seriously misleading for several reasons, and when attempting to establish that transmission occurred between specific people virological evidence should be used with caution and only in conjunction with the clinical and epidemiological evidence.

Viral phylogenetics provides methods for assessing the relations between viruses from different people. A phylogeny is a hypothesis under which we can estimate the probability that viruses from two particular people have a recent common origin, but only in relation to other strains compared. The reliability of the conclusion depends both on the assumptions made in the statistical analysis and the data available for analysis.

Statistical tests (parametric, non-parametric, or Bayesian) are used to evaluate one such hypothesis against another, but if an inaccurate model is specified, or if the most relevant data are not included, these tests can, perhaps counterintuitively, give formal support to an incorrect conclusion. There are serious limitations on what can and cannot be inferred using phylogenetics alone and, in our view, a conviction that relies on such evidence to establish transmission is inherently unsafe. For these reasons, expertise should be sought before undertaking such analyses. ${ }^{7-9}$

The greatest difficulty lies with the nature of the data. Identifying a linkage between viruses from two people on its own says nothing about the direction of transmission (who infected whom?) without allied information for the individuals concerned, and multiple specimens may be needed from before and after infection. Secondly, it is unlikely that all sexual contacts of all HIV infected people will be available for viral testing; indeed some may not be diagnosed. Thus, it is extremely difficult to distinguish a direct transmission between two people from a transmission from a third party to both, or from the first to the second person indirectly through a third. Thirdly, because HIV infected people can be coinfected or superinfected with genetically diverse strains, interpretation of the phylogeny is even more complex. Finally, similarities in two virus genomes may occur as a result of convergent or parallel evolution. An example of such is the independent development of drug resistance mutations, which can erroneously link people with no history of direct contact. ${ }^{10}$ Now that this is known, genetic positions subject to mutation associated with drug resistance can be excluded, but other sources of convergent evolution, such as those that might be produced by the virus evolving to escape the immune system, are not as well characterised.

We therefore advise caution when interpreting such data because the strength of any apparent linkage between viruses will never approach the degree of certainty generally expected of "DNA" data in a criminal court, which juries are more familiar with. Phylogenetic evidence-together with clinical and epidemiological evidence regarding likely duration of infection, sexual history, and other relevant factors-can provide support for linkage between cases but cannot prove transmission.

In this context, the only safe use of virus gene sequences is in circumstances where the genetic differences between viruses are sufficient to make linkage between two people doubtful (or in the case of different HIV-1 subtypes, highly unlikely). This is equivalent to the ability of blood grouping to establish that two samples come from different people (when the blood group differs), but not that they come from the same person (when the blood group is the same).

Despite the difficulty in determining linkage between specific individuals, phylogenetics can provide important new insights in investigations. A recent example is a study of the timing of HIV-1 infections among Libyan children in hospital, which showed that most infections occurred before the arrival of the accused medical workers in the country. ${ }^{11}$ Within the UK, new diagnoses comprise imported infections, many from sub-Saharan Africa, together with a growing number of infections acquired within the UK, overwhelmingly in men who have sex with men. The speed with which imported infections, from Eastern Europe as well as Africa, will lead to ongoing spread within the UK is unknown, as is the future mixing of viruses between different risk groups (those practising heterosexual or homosexual sex).

Molecular epidemiological approaches, allied to existing surveillance of HIV, will allow sensitive real time monitoring of such trends to be established, thus guiding targeted and cost effective public health interventions.

It will be important that sufficient checks and balances are in place to allow full use of such data for public health benefit, without concern that the underlying purpose for identifying possible viral genetic linkage between people will be to support criminal proceedings.

References are on bmj.com 


\section{Pharmacovigilance in developing countries}

\section{Requires collaboration between stakeholders to develop novel models of funding}

\begin{abstract}
Munir Pirmohamed professor of
clinical pharmacology

munirp@liv.ac.uk

Kwame N Atuah postdoctoral

research fellow, Department

of Pharmacology, University of

Liverpool, Liverpool L693GE

Alex N O Dodoo senior

pharmacovigilance scientist,

Department of Pharmacology,

Centre for Tropical Clinical

Pharmacology and Therapeutics,

University of Ghana Medical

School, Korle-Bu Teaching

Hospital, Accra, Ghana

Peter Winstanley professor of

clinical pharmacology, Department

of Pharmacology, Department

of Pharmacology, University of

Liverpool, Liverpool L69 3GE

Competing interests: MP is a

member of the UK Commission

on Human Medicines. PW is the

chairman of the CDA Product

Development Team (MMV, GSK,

and WHO-TDR). ANOD has received

research funding from WHO-TDR.

Provenance and peer review:

Non-commissioned; externally peer

reviewed.
\end{abstract}

BMJ 2007;335:462

doi: 10.1136/bmj.39323.586123.BE
Efforts are increasing to ensure that resource poor countries, which bear almost $90 \%$ of the global disease burden, have access to effective medicines. ${ }^{1}$ As a result, drug companies are facing increased pressure from governments, the World Health Organization, and patient lobby groups to remove legal and financial barriers to access. ${ }^{2}$ However, although these campaigns are necessary and clearly laudable, they are not accompanied by the development or upscaling of processes for monitoring drug safety. Although many drugs have been extensively used and studied in developed countries (thus informing global practice), their safety profile cannot necessarily be generalised to developing countries, where the incidence, pattern, and severity of adverse reactions may differ markedly because of local environmental and genetic influences. ${ }^{3}$

After the thalidomide disaster in the $1960 \mathrm{~s}$, most Western countries developed national pharmacovigilance systems. ${ }^{4}$ These systems use spontaneous reporting or other pharmacoepidemiological methods to systematically collect and analyse adverse events associated with the use of drugs, identify signals or emerging problems, and communicate how to minimise or prevent harm. Although these processes are not perfect, as exemplified by recent problems, ${ }^{5}$ they do provide evidence that can be used to institute regulatory action to protect public health.

At the global level, the WHO programme for international drug monitoring at the Uppsala Monitoring Centre collates adverse drug reaction reports via the national pharmacovigilance centres of the 81 member countries www.who-umc.org). However, currently only six sub-Saharan African countries (South Africa, Zimbabwe, Tanzania, Mozambique, Nigeria, and Ghana) are full members of the programme. In fact, less than $27 \%$ of lower middle income and low income economies have national pharmacovigilance systems registered with the WHO programme, compared with $96 \%$ of the high income countries in the Organisation for Economic Co-operation and Development. The main reasons for this are lack of resources, infrastructure, and expertise. Thus, although access to medicines is increasing in developing countries, there is a danger that their risk benefit profiles in indigenous populations will not be fully monitored and acted upon.

So what can be done to improve drug safety monitoring in developing countries? In the short term, we need to make better use of ongoing or planned studies. The ability to detect an adverse drug reaction depends on its frequency and the total number of people exposed to the drug. ${ }^{6}$ A logical approach would be to encourage collaboration between academic investigators, drug companies, and governments undertaking clinical studies to develop common adverse reaction reporting forms and to deposit the data into a single database.

Similar partnerships could also be established with organisers of public health and drug access campaigns and with regional surveillance systems, such as the East African network for monitoring antimalarial treatment ${ }^{7}$ and the network for assessing health and demography in developing countries. ${ }^{8}$ The operational advantages of this approach are that data can be obtained from a range of studies and that pre-existing manual and technical infrastructures can be used to acquire the data. This would provide demographically relevant data from large (and less homogeneous) populations in a structured and systematic fashion, and these data could then be used to identify warning signals.

Individual investigators would still own their data and publish results of their trials, but the pooling of data on adverse drug reactions would add value to ongoing studies. This has already happened on a small scale. For example, an increased risk of serious neurological reactions was identified in people taking ivermectin who were infected with Loa loa before treatment started. ${ }^{9}$ Such pooling of data needs to be increased and considered for all drug classes within a formulary.

What role should the drug industry have in promoting pharmacovigilance? The current model for drug development in resource poor settings depends on publicprivate partnerships, such as the Medicines for Malaria Venture. These partnerships should be encouraged to continue beyond the point of obtaining a drug licence to developing a proactive phase IV programme. Such a programme could be designed to show the effectiveness of the drug in a real world situation, and through this obtain safety data in much larger cohorts of patients. A few examples of this approach already exist in Africa, ${ }^{10}$ but these need to become the norm rather than the exception.

In the long term, every country should develop its own national pharmacovigilance system, which contributes to a global database such as that held by the Uppsala Monitoring Centre. This will need an extensive infrastructure, however, which would be costly. In a climate where health resources are limited, funding a pharmacovigilance system will come second to other competing priorities such as implementing a new vaccine programme. The funding model for pharmacovigilance activities in the United States recently advocated by the Institute of Medicine ${ }^{11}$ is unlikely to work in developing countries if it increases drug costs, as this defeats the aim of increasing access to medicines. No easy answers are available, but WHO needs to lead a dialogue between the major stakeholders with the aim of developing a novel funding model that supports pharmacovigilance activities in developing countries. The lack of local expertise in pharmacovigilance could be tackled through developing exchange programmes with the major drug regulatory agencies and sharing of best practices.

References are on bmj.com 